\title{
Giant Gastric Ulcers: An Unusual Culprit
}

\author{
Aamer Abbass $^{1} \cdot$ Sameen Khalid ${ }^{1} \cdot$ Vaishnavi Boppana ${ }^{2} \cdot$ Joshua Hanson $^{3} \cdot$ Henry Lin $^{1,2} \cdot$ Denis McCarthy $^{1}$
}

Published online: 2 September 2020

(c) Springer Science+Business Media, LLC, part of Springer Nature 2020

\begin{abstract}
Mycophenolate Mofetil (MMF) is routinely used immunosuppressant in solid organ transplantation is commonly associated with several gastrointestinal (GI) side effects. Here we present a case of giant gastric ulcer of $5 \mathrm{~cm}$ from MMF use post cardiac transplant.

Case Description A 56-year-old male with history of severe ischemic cardiomyopathy post heart transplant was on immunosuppression with MMF, tacrolimus and prednisone for 5 months. He presented with severe epigastric pain and intermittent episodes of melena for 1 month. His pain radiated to back that is worsened with eating. Associated with loss of appetite, vomiting and 16-pound weight loss in 3 months. He never smoked, drank alcohol or used over the counter pain medications. He was profoundly anemic requiring blood transfusions. EGD performed demonstrated very large clean-based ulcer of $5 \mathrm{~cm}$ diameter in the body, smaller ulcer of $8 \mathrm{~mm}$ diameter in pre-pyloric region and 5-10 small aphthous ulcers in the gastric body and fundus. Gastric biopsies taken from the ulcer were negative for Helicobacter pylori, cytomegalovirus and malignancy. Flexible sigmoidoscopy revealed non-bleeding inflamed internal hemorrhoids. Consequently, MMF was discontinued and switched to azathioprine. He was treated with twice daily proton pump inhibitor therapy with resolution of abdominal pain, improved appetite and weight gain.

Discussion MMF is well known for common GI side-effects such as nausea, diarrhea, vomiting, ulcers, abdominal pain and rarely gastrointestinal bleeding. Few studies reported 3 to $8 \%$ incidence of ulcer perforation and GI bleeding within 6 months. Risk of gastroduodenal erosions is nearly 1.83 times for MMF, with the highest lesions associated with MMFtacrolimus-corticosteroid combination treatment as seen in our patient. Hypothesis is that GI tract is vulnerable because of dependence of enterocytes on de novo synthesis of purines, which is disrupted by MMF. Typically, upper GI mucosal injuries of mucosal irritation leading to esophagitis, gastritis and/or ulcers are seen. Endoscopy is both diagnostic and therapeutic if bleeding gastric ulcers are noted. Minor complications improve with reduction of drug dose or use of enteric coated preparation if feasible. Discontinuation of the drug is main stay in the management of MMF related ulcer disease. Simple medical treatment with either H2-receptor antagonists, proton-pump inhibitors, coating agents, prostaglandins or combination has proven effective in most cases. Considering excellent results with medical management of ulcer, role of surgery is limited.
\end{abstract}

Keywords Gastric ulcer · Cardiac transplant · Mycophenolate mofetil $\cdot$ Mycophenolic acid $\cdot$ Foveolar hyperplasia

\section{Case}

Aamer Abbass

aabbass@salud.unm.edu

1 Division of Gastroenterology and Hepatology, University of New Mexico, MSC 10-5550, Albuquerque, NM 87131, USA

2 Department of Internal Medicine, University of New Mexico School of Medicine, Albuquerque, NM, USA

3 Department of Pathology, University of New Mexico School of Medicine, Albuquerque, NM, USA
A 56-year-old male with a long history of gastroesophageal reflux disease, multi-vessel coronary artery disease, and ischemic cardiomyopathy with a left ventricular ejection fraction of $15 \%$, underwent orthotopic cardiac transplantation. Eight weeks afterward, he developed multiple painful oral aphthous ulcers while receiving mycophenolate mofetil (MMF) $1000 \mathrm{mg}$ twice a day, tacrolimus $3 \mathrm{mg}$ twice a day, prednisone $30 \mathrm{mg}$ once a day, aspirin $81 \mathrm{mg}$, and pantoprazole $40 \mathrm{mg}$ once a day for "gastric ulcer prophylaxis." With aphthous ulcers and a mild leucopenia of $3100 / \mu \mathrm{L}$ (normal: 
$4000-11,000 / \mu \mathrm{L})$, with an otherwise normal white cell differential count, MMF was reduced to $500 \mathrm{mg}$ twice daily and prednisone to $20 \mathrm{mg}$ daily, while continuing pantoprazole $40 \mathrm{mg}$ daily, with oral lidocaine solution added as needed for symptom relief. Though the oral ulcers resolved within a few weeks, 12 weeks post-transplantation, he developed the rapid onset of epigastric pain after every meal, associated with nausea, vomiting, and a $20 \mathrm{lb}$. weight loss, the latter due to his oral intake being decreased for fear of aggravating the pain. The dose of pantoprazole was increased to $40 \mathrm{mg}$ b.i.d., and prednisone dose was decreased to $10 \mathrm{mg}$ daily. Despite these changes, epigastric pain continued and he was unable to tolerate an adequate oral intake. A few weeks later, he was evaluated in the emergency department with melena, worsening epigastric pain, loss of appetite, and generalized weakness for 2 days. On examination, he appeared pale, with a blood pressure of $154 / 76 \mathrm{~mm} \mathrm{Hg}$, a heart rate of 108 beats/min, and epigastric tenderness on palpation, but with no signs of peritonitis, such as rebound tenderness, marked guarding or diffuse abdominal rigidity. Bowel sounds were normal. Initial laboratory tests (with normal ranges) showed a blood hemoglobin of $9.6 \mathrm{~g} / \mathrm{dL}$, fallen from a baseline 2 weeks previously of $14.2 \mathrm{~g} / \mathrm{dL}(13.5-17.7 \mathrm{~g} / \mathrm{dL})$, a mean corpuscular volume (MCV) $89 \mathrm{fL}(80-100 \mathrm{fL})$, a red cell distribution width (RDW) of $13 \%$ (11-14.5\%), a platelet count of $443,000 / \mu \mathrm{L}(150,000-400,000 / \mu \mathrm{L})$, and a total white blood cell count (WBC) of 3000/ $\mu \mathrm{L}(4000-11,000 /$ $\mu \mathrm{L})$. Iron profile showed serum iron of $28 \mathrm{ug} / \mathrm{dl}(65-170 \mathrm{ug} /$ $\mathrm{dl})$, total iron binding capacity of $231 \mathrm{ug} / \mathrm{dl}(250-450 \mathrm{ug} / \mathrm{dl})$, iron saturation of $12 \%$ (20-55\% normal limits), and serum ferritin (non-fasting) of $2585 \mathrm{ng} / \mathrm{ml}(30-530 \mathrm{ng} / \mathrm{ml})$. The low serum iron, low iron saturation, and thrombocytosis suggested the presence of iron deficiency anemia, despite the high serum ferritin which is as yet unexplained. Other blood tests were all normal. Computerized tomographic angiography (CTA) scans of the abdomen and pelvis (with and without contrast) showed no occlusion within celiac or mesenteric vasculature, and the hepatobiliary system and spleen within normal limits. Promptly hospitalized, he underwent esophagogastroduodenoscopy (EGD). While the esophagus was normal, EGD showed two large ulcer craters ( $1 \mathrm{~cm}$ and $5 \mathrm{~cm}$, respectively) in the gastric antrum, with heaped up erythematous edges, and multiple (about $10)$, small $(<5 \mathrm{~mm})$, non-bleeding aphthous ulcers in the corpus: No abnormality was seen in the duodenum. Multiple biopsies were taken from the ulcer edges, with additional random biopsies from the adjacent gastric mucosa. Histologic examination of these showed gastric antral and oxyntic mucosa with foci of erosion, and reactive foveolar hyperplasia. Immunostains for CMV and Helicobacter pylori, and GMS stain for fungal elements were all negative; the biopsies showed no intestinal metaplasia, dysplasia, or malignant change. After consultation with the transplantation team and pathologist, the MMF was discontinued and the patient's therapy was changed to azathioprine with continuation of tacrolimus, prednisone, and pantoprazole. Sucralfate solution $1 \mathrm{~g}$ four times a day was started. A day after the EGD, he was able to tolerate a full liquid diet and was discharged home.

Despite attempts to restore a normal diet, his epigastric pain and nausea persisted and 3 weeks later, he developed recurrent melena and was readmitted to hospital. Physical examination showed generalized pallor, with normal vital signs but focal epigastric tenderness. Laboratory investigations showed a blood hemoglobin of $7.7 \mathrm{~g} / \mathrm{dL}$, reduced from $9.6 \mathrm{~g} / \mathrm{dL}$ during his last admission, an MCV of $90 \mathrm{fL}$, an RDW of $17 \%$, a WBC count of 6100 , and a platelet count of $316,000 / \mu \mathrm{L}$. A repeat EGD showed (Fig. 1) persistence of a large 5-cm gastric antral ulcer, a second $2-\mathrm{cm}$ antral ulcer, and multiple smaller ulcers in the gastric body with
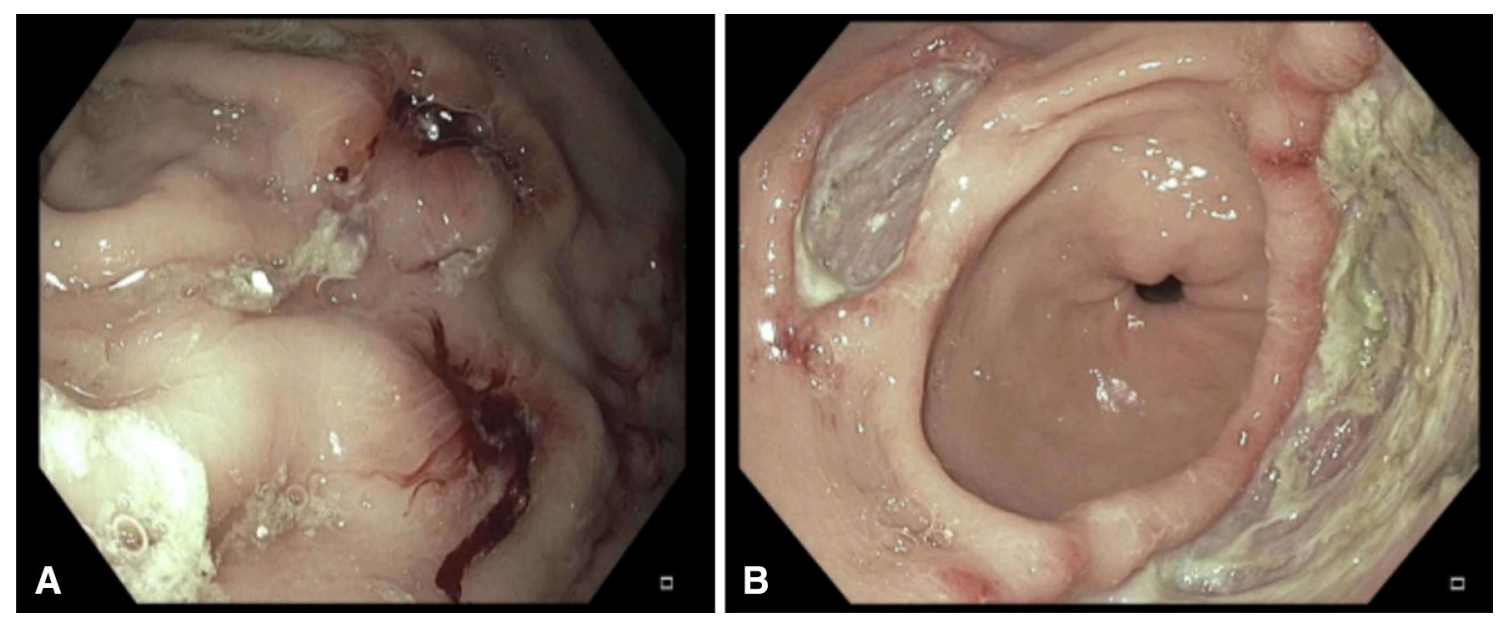

Fig. 1 A Two small ulcers in gastric corpus with adherent clots. B Two large gastric ulcers in gastric antrum 
adherent clots, diffuse mucosal congestion, and erythema with normal esophagus and duodenum. Multiple biopsies were taken from both gastric ulcers, and from numerous areas of gastric mucosa with the biopsy specimens placed in separate labeled jars. Histologic examination of the gastric ulcers showed erosive injury, with acute and chronic inflammation in the lamina propria. Furthermore, the biopsies also showed foveolar hyperplasia and increased cellular apoptosis in the gastric glands and the ulcers, consistent with mycophenolate-induced mucosal injury (Fig. 2), although such apoptotic changes due to mycophenolate are best documented in the colon. There was no evidence of malignant disease. As expected, repeat testing for Helicobacter pylori, $\mathrm{CMV}, \mathrm{EBV}$, and fungi remained negative.

Due to his inability to tolerate oral feeding, the patient was started on feeding via a nasogastric tube together with intravenous hydration. His medications included intravenous pantoprazole $40 \mathrm{mg}$ twice a day and oral liquid sucralfate $1 \mathrm{gm}$ four times a day. He responded well with, reduced epigastric pain, increased ability to tolerate an oral diet, and resultant weight gain. He was discharged home on the above regimen as well as tacrolimus, azathioprine, low-dose prednisone, and $81 \mathrm{mg} / \mathrm{d}$ of aspirin. At the gastroenterology outpatient clinic 10 weeks after discharge, he was doing well, with resolution of abdominal pain, and nausea and diminishing anemia (hemoglobin of $10 \mathrm{~g} / \mathrm{d}$ ). He had not received iron therapy. Sucralfate was discontinued, and pantoprazole was reduced to once a day. However, due to the COVID-19 pandemic, a repeat EGD in order to assess ulcer healing has not been performed to date, nor has his high serum ferritin been investigated, but symptomatically and clinically the patient is doing well.

\section{Discussion}

The finding of foveolar hyperplasia $(\mathrm{FH})$ in a gastric biopsy, as in this case, is the cardinal feature of a reactive gastritis resulting from chemical injury from the lumen by, for example, duodeno-gastric bile reflux or the presence aspirin or a non-steroidal anti-inflammatory drug (NSAID), but it can be caused by many kinds of mucosal injury, rarely including that due to acid-pepsin. The use of low (baby) doses of aspirin may be associated with foveolar hyperplasia, or GI bleeding from preexisting lesions [1], but not with proven gastric ulcers; the dose of aspirin statistically significantly associated with the development of gastric ulcer is 22 tablets/week [2]. While low doses of aspirin taken chronically have been found to impair gastric prostaglandin synthesis and cause gastric erosive injury, in patients in whom $H$. pylori infection was not excluded, there is no evidence identifying low dose of aspirin as a cause of giant gastric ulcers [3].

While steroids are no longer believed to cause gastric ulcers, rarely giant gastric ulcers have been attributed to sustained chronic ischemia. In this case, however, CTA revealed normal gastric and mesenteric vessels, and the patient was not known to have been hypotensive at any time: Transient hypotension would not cause focal ischemic lesions limited to the stomach.

Post-transplant transthoracic echocardiogram showed normal left ventricular ejection fraction and diastolic function. The presence of FH should immediately alert the clinician to the patient's use of some toxic drug or other chemical agent. In this case, the presence of apoptosis, in
Fig. 2 Pathology of gastric mucosa near one of the large ulcers. The pattern is erosive gastropathy with increased lamina propria acute and chronic inflammation. In addition, there is increased apoptotic activity in the gastric glands (apoptotic bodies are highlighted by the arrows in the image)

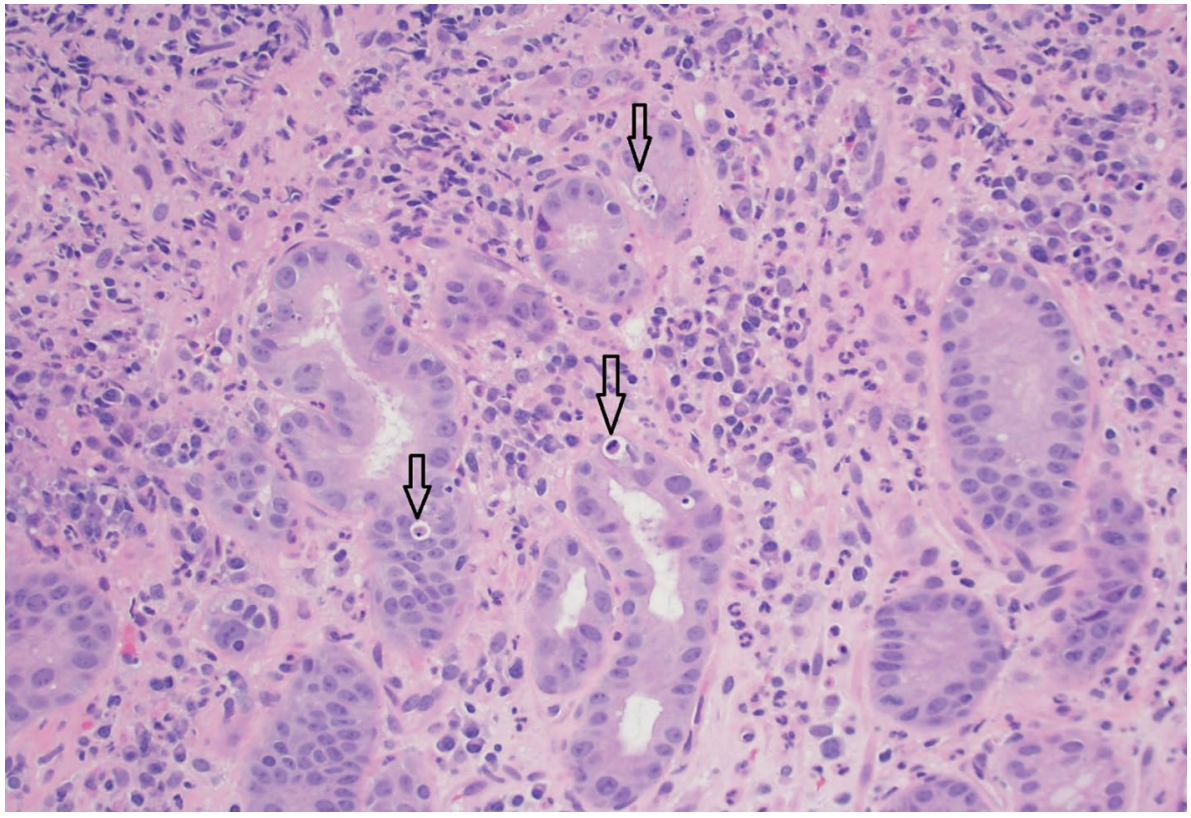


the absence of $H$. pylori infection or exposure to therapeutic doses of aspirin, pointed strongly to MMF as the culprit. The essence of treatment of injury due to MMF is elimination of exposure to the toxic agent. Therapies with compounds such as proton pump inhibitors or sucralfate have no reported value, and proton pump inhibitors, by affecting the microbiome (see below), could have adverse effects.

In the management of patients who receive an organ transplant, mycophenolate mofetil (MMF) is commonly used as an immunosuppressive drug to prevent rejection of the donated organ [4-6]. It is a synthetic ester derived from mycophenolic acid, a compound naturally produced by Penicillium brevicompactum or related fungi, and was first isolated in 1898 [6, 7]. Two preparations of mycophenolic acid are available in the USA, MMF (Cellcept, Roche Pharmaceuticals) and mycophenolate sodium (Myfortic, Novartis Inc.). Myfortic is an enteric-coated, delayed-release formulation and is therefore absorbed in the small intestine, whereas MMF is not enteric-coated but is an immediate-release formulation which is hydrolyzed by tissue and plasma esterases to the active metabolite mycophenolic acid (MPA) that is absorbed in the stomach and proximal small intestine [6-8]. Despite these differences, the efficacies of the two compounds are similar in preventing transplant rejection and in their side effect profiles.

The mechanism of action of MPA is inhibition of inosine monophosphate dehydrogenase (IMPDH), a rate-limiting enzyme, in the pathway of purine synthesis $[6,7,9-11]$. MMF is an anti-metabolite that is a highly selective, noncompetitive, and reversible inhibitor of IMPDH. The immunosuppressive property of MPA is due to its inhibition of de novo purine synthesis [6, 7, 9-11]. B and T lymphocyte functions are $90 \%$ dependent on this pathway. In T and B lymphocytes, the de novo pathway involves conversion of 5-phosphoribosyl-1-pyrophosphatase to inosine monophosphate, which is then converted to guanosine monophosphate by the enzyme IMPDH, a rate-limiting step. Guanosine triphosphate is subsequently produced and contributes to DNA synthesis [12]. Thus, by inhibiting de novo purine synthesis in lymphocytes, MMF suppresses cytotoxic $\mathrm{T}$ lymphocyte activity responsible for cellular rejection, and of the B lymphocytes that contribute to antibody-mediated immunosuppression. Since enterocytes are 50\% dependent on this same pathway growth and development, MPA injures the epithelium by inhibiting the proliferation of all enterocytes [6].

Owing to its immunosuppressant effectiveness, the use of MMF has revolutionized the field of transplantation, significantly reducing early graft rejection, promoting long-term graft survival, and possessing an acceptable level of toxicity [7]. Despite a generally favorable safety profile, MMF has been associated with a multitude of adverse effects occurring throughout the gastrointestinal (GI) tract [10]. In 40-50\% of patients, GI intolerance of MMF is the leading cause of dosage changes and interruptions or discontinuations of immunosuppressive therapy [13]. The incidence of MPA-related GI adverse effects varies from 45 to $80 \%$ [13-16]. Common GI symptoms include abdominal pain, nausea, vomiting, diarrhea, constipation, anorexia, ulcers, and dyspepsia [4, 17], among which diarrhea is most frequently reported the adverse effect [5]. MMF has also been reported to cause topical mucosal irritation and damage, leading to esophageal, gastric, and duodenal ulcers, and a reactive gastritis similar to that caused by NSAIDs [5]. The incidence of dyspepsia with MMF or NSAIDs is reported to be approximately $40-45 \%$, but the reported incidence of ulcer perforation and GI bleeding is higher for MMF (3-8\% within 6 months of initiation) compared with NSAIDs (0.5-4\% within 1 year) ([18]: unsupported statement).

Furthermore, in a study looking at GI toxicity in postrenal transplant patients on various combinations of immunosuppressive therapies including MMF, the risk of gastroduodenal erosions was elevated for MMF as an independent risk factor with odds ratio of 1.83 (1.02-3.29, $P=0.043)$ when corrected for the presence of other risk factors including Helicobacter pylori and pulsed dose steroids. In the same study, the highest incidence of gastric erosion was observed in MMF-tacrolimus-corticosteroid combination treatment as used in this case [19]. Fewer studies reported a 3-8\% incidence of ulcer perforation and GI bleeding within 6 months using MMF alone [20]. Although the finding of a large gastric ulcer, $5 \mathrm{~cm}$ in diameter, and a smaller crater $2 \mathrm{~cm}$ in diameter in the present case is rare, Chang et al. described a similar case of non-healing gastric and duodenal ulcers due to MMF use in a 36-year-old female with a history of familial intrahepatic cholestasis following liver transplantation [4]. Although upper GI toxicity is a well-known complication of MMF, the literature describing attributed pathologic findings the in the upper GI tract is sparse. The first description of histologic features of MPA-induced damage in the upper GI tract was from Ducloux et al. in 1998, who described villous blunting and crypt hyperplasia in the duodenum of a patient taking MMF after kidney transplantation [21]. The effects on the upper GI tract of exposure to MMF are similar to those of NSAIDs and include local mucosal irritation with ulcerative esophagitis, reactive gastritis, and gastric and duodenal ulcers. In the lower GI tract, the anti-metabolite effects of MMF give rise to the graft versus host disease (GVHD)-like changes of an MMF-induced colitis [5]. Parfitt et al., who described the pathologic features of MMF toxicity in both the upper and lower GI tract, correlated them with clinical and endoscopic findings. They noted the following features in the upper GI tract: active esophagitis with ulceration or erosion; chemical gastritis and Crohn-like features in the 
stomach; and GVHD-like changes and Crohn-like feature in the duodenum. They also observed that MMF-induced injury of the upper GI tract leads to an increase in crypt epithelial apoptosis similar to mild or grade I GVHD-like injury. Nguyen et al. [6] provided the apoptotic count guidelines that help the recognition of MMF-induced injury in the upper GI tract. The time interval between transplantation and endoscopy ranged from 1 month to more than 10 years, indicating that the time interval for MPA to cause GI damage and patient symptoms is variable [6]. In their study, $28 \%$ (5 out of 18) gastric biopsies showed abnormal apoptotic counts of $\geq 3 / 100$ glands [6]. They observed abnormal apoptotic counts not only in a background of otherwise normal mucosa, but also in injured mucosa that demonstrated additional changes: chronic duodenitis, acute duodenitis, duodenal erosion, celiac changes, chemical gastropathy, active chronic gastritis, or gastric erosion [6].

The mechanism of MMF-related GI toxicity is still not fully clear. The GI tract is quite vulnerable since enterocytes are $\sim 50 \%$ dependent on the de novo synthesis of purines that is disrupted by MPA with consequent mucosal damage $[11,16]$. Some researchers believe in a local and direct toxicity of MPA on intestinal mucosa. That acyl-glucuronide, a byproduct of MPA metabolism, in higher concentrations has direct tissue toxicity, supports this concept [22]. A histologically abnormal apoptotic pattern and ballooning degeneration, seen on gastric and duodenal biopsies, are suggestive of MMF-induced gastroduodenitis [6, 23, 24]. For over 20 years, it has been known that tissues exposed to MPA exhibited impaired healing, associated with an unusual dysfunction of the fibroblast cytoskeleton which contained less vinculin, actin, and tubulin than seen in control tissues.

Approximately $5 \%$ of EGDs in totally asymptomatic normal subjects reveal erosions, indicating that some epithelial breakage is part of normal wear and tear, but this is balanced by the countering effects of repair processes. Due to interactions with inflammatory mediators, tissues exposed to MPA have impaired healing, arising from inhibition of the inflammatory and cellular phases of the process [12]. MMF also impairs the healing of colonic anastomoses early in the postoperative period [25]. Evaluating the effect of MMF on the healing of left-sided colonic anastomoses in rats, more extensive inflammation was observed in MMF-treated animals than in controls [26]. This study concluded that MMF inhibits injuryinduced reparative proliferation of colonic mucosal cells [26]. In a similar study conducted by Sikas et al., MMF weakened the integrity of colonic anastomoses [25]. They found that MMF has a negative effect on macrophages and TGF- $\beta 1$ expression with reduced accumulation of collagen at the weakened anastomotic site [25]. Roos et al. showed that MMF exerts direct effects on fibroblast extracellular matrix remodeling, by inhibiting collagen gene expression, extracellular matrix contraction, and fibroblast migration [27]. In fibroblasts exposed to pharmacological doses of MPA, Morath et al. described downregulation of cytoskeletal proteins such as vinculin, actin, and tubulin [28], with resultant rearrangement of the cytoskeleton in MPA-treated fibroblasts, thought to impair healing [28]. In addition to inhibiting $\mathrm{T}$ and $\mathrm{B}$ cell proliferation, MMF inhibits proliferation of other cell types including smooth muscle cells, renal tubular cells, mesangial cells, fibroblasts, and enterocytes [28, 29], and in addition inhibits fibroblast migration.

In addition to these observations, studies in mice have shown that intestinal microbiota contribute to the pathogenesis of MMF-induced GI toxicity [30]. Mice fed MMF in chow exhibited changes in the composition of the intestinal microbiome, including loss of microbial diversity and expansion of Proteobacteria, Escherichia, and Shigella, and increased expression of lipopolysaccharide, indicating an increased preponderance of Gram-negative bacteria. Mice treated with MMF suffered from significant weight loss and marked colonic inflammation, whereas germ-free mice treated with MMF did not, indicating that MMF-related GI toxicity is dependent on the intestinal flora. Furthermore, antibiotics could both prevent and reverse the MMF-induced GI toxicity: A cocktail of antibiotics ampicillin, metronidazole, neomycin, and vancomycin given for 2 weeks prior to start of MMF prevented the development of GI toxicity, and given 8 days after MMF administration reversed the toxicity, suggesting an importance of the microbiome in modifying the GI toxicity due to MMF. This may well occur in human solid organ recipients and remains a potential area for further research.

EGD with biopsies is indicated in any MMF user with pain or dyspepsia. Gastric ulcers and gastritis pose diagnostic challenges, especially in NSAID users, but the presence of foveolar hyperplasia and apoptosis greatly enhance the likelihood of damage by an ingested agent and reduce the likelihood of acid-peptic injury and Helicobacter pylorirelated chronic active gastritis. CMV, commonly encountered in immunosuppressed subjects, may be present in any ulcerated lesion, regardless of cause; only when the virus is demonstrated in endothelial cells or macrophages may it be regarded as the significant pathogen. In cases with GI bleeding, EGD may be both diagnostic and therapeutic. In some cases, minor upper GI symptoms may improve with reduction of drug dose, or with use of the enteric-coated preparation [31]. Nevertheless, when the mucosa is demonstrably injured, e.g., demonstrating MMF-related ulcer disease or gastritis, discontinuation of drug use is the mainstay of management [11]. Reduction of the dose, or avoidance of drug use until wound healing is complete, has also been advocated. Treatments with H2-receptor antagonists, proton pump inhibitors, coating agents such as sucralfate, or 
prostaglandins have not been shown to be of benefit although a liquid antacid may be used for symptom relief.

\section{Key Points}

- MMF is an immunosuppressive drug following solid organ transplantation. It is a prodrug that is hydrolyzed to MPA, its active ingredient, which acts by reversibly inhibiting IMPD, a rate-limiting enzyme in de novo purine synthesis.

- GI toxicity with the use of MMF may vary from selflimiting symptoms such as nausea, vomiting, diarrhea, abdominal pain to more severe complications, e.g., ulcers resulting in bleeding and/or perforation.

- The dependency of most enterocytes on de novo purine synthesis and the direct toxicity of MPA on GI mucosae, with disruption of mucosal barriers and impaired processes of repair, are the presumed pathogenic mechanisms: These changes are affected by intestinal microbiota.

- EGD with biopsies is usually diagnostic and may be therapeutic in patients with persistent or severe symptoms.

- Dose modifications including decrease in the dose, or a drug-free period, may improve mild-to-moderate symptoms, but withdrawal of MMF is recommended in patients with demonstrable mucosal injury or ulceration.

\section{References}

1. McCarthy DM. Ulcers Helicobacter pylori infection, platelets and complications of non-steroidal anti-inflammatory drugs: What are the connections? Eur J Surg. 2002;587:89-99.

2. Graham DY, Smith JL. Aspirin and the stomach. Ann Int Med. 1986;104:390-398.

3. Cryer B, Feldman M. Effects of very low dose daily, long-term aspirin therapy on gastric, duodenal, and rectal prostaglandin levels and on mucosal injury in healthy humans. Gastroenterology. 1999;117:17-25.

4. Chang VC, Mai D, Park M, Lee D, Samarasena J. Mycophenolate mofetil-induced non-healing gastric and duodenal ulcers. Am J Gastroenterol. 2018;113:S1089-S1090.

5. Parfitt JR, Jayakumar S, Driman DK. Mycophenolate mofetilrelated gastrointestinal mucosal injury: variable injury patterns, including graft-versus-host disease-like changes. Am J Surg Pathol. 2008;32:1367-1372.

6. Nguyen T, Park JY, Scudiere JR, Montgomery E. Mycophenolic acid (Cellcept and Myofortic) induced injury of the upper GI tract. Am J Surg Pathol. 2009;33:1355-1363.

7. Sollinger HW. Mycophenolates in transplantation. Clin Transpl. 2004; 18:485-492.

8. Staatz CE, Tett SE. Pharmacology and toxicology of mycophenolate in organ transplant recipients: an update. Arch Toxicol. 2014;88:1351-1389.
9. Allison AC, Eugui EM. Immunosuppressive and other antirheumatic activities of mycophenolate mofetil. Agents Actions Suppl. 1993;44:165-188.

10. Zwerner J, Fiorentino D. Mycophenolate mofetil. Dermatol Therapy. 2007;20:229-238.

11. Behrend M. Adverse gastrointestinal effects of mycophenolate mofetil. Drug Saf. 2001;24:645-663.

12. Bootun R. Effects of immunosuppressive therapy on wound healing. Int Wound J. 2013;10:98-104.

13. Bunnapradist S, Ambühl PM. Impact of gastrointestinal-related side effects on mycophenolate mofetil dosing and potential therapeutic strategies. Clin Transpl. 2008;22:815-821.

14. Jia Y, Wang R, Li L, Zhang Y, et al. Sites of gastrointestinal lesion induced by mycophenolate mofetil: a comparison with enteric-coated mycophenolate sodium in rats. BMC Pharmacol Toxicol. 2018;19:39.

15. Díaz B, González Vilchez F, Almenar L, Delgado JF, et al. Gastrointestinal complications in heart transplant patients: MITOS study. Transpl Proc. 2007;39:2397-2400.

16. Arns W. Noninfectious gastrointestinal (GI) complications of mycophenolic acidl therapy: a consequence of local GI toxicity? Transpl Proc. 2007;39:88-93.

17. Halloran P, Mathew T, Tomlanovich S, Groth C, et al. Mycophenolate mofetil in renal allograft recipients: a pooled efficacy analysis of three randomized, double-blind, clinical studies in prevention of rejection. The International Mycophenolate Mofetil Renal Transplant Study Groups. Transplantation. 1997;63:39-47.

18. Bjarnason I. Enteric coating of mycophenolate sodium: a rational approach to limiting topical gastrointestinal lesions and extend the therapeutic index of mycophenolate. Transpl Proc. 2001;33:3238-3240.

19. Telkes G, Peter A, Tulassay Z, Asderakis A. High frequency of ulcers, not associated with Helicobacter pylori, in the stomach in the first year after kidney transplantation. Nephrol Dial Transpl. 2011;26:727-732.

20. Ponticelli C, Passerini P. Gastrointestinal complications in renal transplant recipients. Transpl Int. 2005;18:643-650.

21. Ducloux D, Ottignon Y, Semhoun-Ducloux S, et al. Mycophenolate mofetil-induced villous atrophy. Transplantation. 1998;66:1115-1116.

22. Staatz CE, Tett SE. Clinical pharmacokinetics and pharmacodynamics of myco-phenolate in solid organ transplant recipients. Clin Pharmacokinet. 2007;46:13-58.

23. Berribi C, Loirat C, Jacqz-Aigrain E. Mycophenolate mofetil may induce apoptosis in duodenal villi. Pediatr Nephrol. 2000;14:177-178.

24. Wong NA. Gastrointestinal pathology in transplant patients. Histopathology. 2015;66:467-479.

25. Sikas N, Imvrios G, Takoudas D, Gakis D, Papanikolaou V. Mycophenolate mofetil impairs the integrity of colonic anastomosis. J Surg Res. 2006;134:168-172.

26. Zeeh J, Inglin R, Baumann G, Dirsch O, et al. Mycophenolate mofetil impairs heal-ling of left-sided colon anastomoses. Transplantation. 2001;71:1429-1435.

27. Roos N, Poulalhon N, Farge D, Madelaine I, et al. In vitro evidence for a direct anti fibrotic role of the immunosuppressive drug mycophenolate mofetil. J Pharmacol. 2007;321:583-589.

28. Morath C, Zeier M. Review of the antiproliferative properties of mycophenolate mofetil in non-immune cells. Int J Clin Pharmacol Ther. 2003;41:465-469.

29. Morath C, Schwenger V, Beimler J, Mehrabi A, et al. Antifibrotic actions of mycophenolic acid. Clin Transpl. 2006;20:25-29.

30. Flannigan KL, Taylor MR, Pereira SK, Rodriquez-Arguello J, et al. An intact microbiota is required for the gastrointestinal 
toxicity of the immunosuppressant mycophenolate mofetil. $J$ Heart Lung Transpl. 2018;37:1047-1059.

31. Suwelack B, Gabriels G, Volmer S, Hillebrand U, et al. Resolution of severe MMF-related gastrointestinal adverse events following conversion to enteric-coated mycophenolate sodium. Transplantation. 2005;79:987-988.
Publisher's Note Springer Nature remains neutral with regard to jurisdictional claims in published maps and institutional affiliations. 